\title{
SIMULATION OF MIXED CONVECTIVE HEAT TRANSFER USING LATTICE BOLTZMANN METHOD
}

\author{
A. R. M. Rosdzimin ${ }^{1}$, S. M. Zuhairi ${ }^{2}$ and C. S. N. Azwadi ${ }^{3}$ \\ ${ }^{1}$ Department of Mechanical Engineering, Faculty of Engineering \\ Universiti Pertahanan Nasional Malaysia \\ Kem Sg. Besi, 57000, Kuala Lumpur, Malaysia \\ Phone:+603-90514500, Fax:+603-90514450 \\ Email: rosdzimin@upnm.edu.my \\ ${ }^{2}$ Faculty of Mechanical Engineering, Universiti Malaysia Pahang, \\ Pekan Campus, Kuantan, Pahang, Malaysia \\ ${ }^{3}$ Faculty of Mechanical Engineering, Universiti Teknologi Malaysia \\ 81300, Skudai, Johor, Malaysia
}

\begin{abstract}
In this paper, mixed (forced-natural) convective heat transfer around a heated square cylinder located inside a lid driven cavity has been studied numerically using the lattice Boltzmann method in the range of $100 \leq \mathrm{Re} \leq 1000$ with the corresponding Richardson number $0.01 \leq \mathrm{Ri} \leq 10$. The double-population lattice Boltzmann formulation is used as the governing equation. Two dimensional nine-velocity models are used for the computation of the velocity field while a four-velocity model is used for the computation of the temperature field. We found that the combination of nine- and fourvelocity models can be applied to the calculation without losing its accuracy. The results are presented in the form of streamline and isotherm plots as well as the variation of local Nusselt number at the top surface of the heated square. The computational results demonstrate that the flow pattern, formation of vortex and also the Nusselt number are influence by the Reynolds number and Richardson number.
\end{abstract}

Keywords: Double-population, thermal lattice Boltzmann, mixed convection.

\section{INTRODUCTION}

The study of flow pattern and heat transfer from a heated body, especially natural convective heat transfer, inside an enclosure, have been carried out by a large number of researchers for a long time. Most of these studies were concerned with the case of a circular cylinder inside a square enclosure. Excellent reviews of this topic were written by Kim et al. (2008) and Angeli et al. (2008). In contrast to the overwhelming number of publications on natural convective heat transfer from a heated circular cylinder, the mixed convective heat transfer problem from a heated body has not been investigated to the same extent, although it plays a dominant role in many engineering applications such as the cooling of electronic equipment, nuclear and chemical reactors, thermal storage system, etc.(for details, Arnab et al., 2006and Sohel et al., 2002). Multiple studies involving heat transfer mechanisms and lid-driven cavity at different cavity configurations, with various fluids and imposed temperature gradients have been continually published in the literature (Oztop and Dagtekin, 2004;Abdalla et al., 2007; Abdelkhalek 2008 and Sharif 2007). 
Ouertatani et al. (2009) simulated three dimensional mixed convection in a double lid driven cubic cavity at various Reynolds numbers and Richardson numbers. These author found that heat transfer improved about $76 \%$ at the particular combination of $\mathrm{Re}=400$ and $\mathrm{Ri}=1$. The fluid flow and heat transfer induced by the combined effects of the mechanically driven lid and the buoyancy force within rectangular enclosures were investigated by Waheed (2009). An investigation at various Prandtl numbers, Richardson numbers and aspect ratios at Reynolds number 100 was simulated by this author. The results showed that fluid flow and energy distribution within the enclosures and heat flux on the heated wall are enhanced by the increase in the Richardson number. Numerical simulation of unsteady mixed convection in a driven cavity using an externally excited sliding lid were studied by Khanaferet al. (2007). These authors examined the fluid flow and heat transfer characteristics in the domain of the Reynolds number, Grashof number and the dimensionless lid oscillation frequency such that: $10^{2}<$ $\operatorname{Re}<10^{3}, 10^{2}<$ Gre $<10^{5}$ and $0.1<\omega<5$. They found that the energy transport process and drag force behaviour depending on the conduct of the velocity cycle were either enhanced or retarded by the Reynolds number and Grashof number. Moreover, relatively small lid oscillation values are found to constrain the lid associated motion to a shallow depth from the sliding lid plane.

Oztop et al. (2009) showed that thermal conductivity becomes insignificant for small values of the diameter of the circular body, by studying the fluid flow due to combined convection in a lid-driven enclosure having a circular body at wide ranges of Richardson numbers, diameters of inner cylinder and centre and location of the inner cylinder. They also concluded that the most effective parameter on the flow field and temperature distribution is the orientation of the moving lid. However, there is little information about the flow pattern and heat transfer mechanism when a heated square cylinder is placed within a cooled square lid driven enclosure. The present paper will investigate how the value of the Richarson number and Reynolds number relative to the local Nusselt number. Simulation results in a range of Richardson number between $0.01 \leq \mathrm{Ri} \leq 10$ and Reynolds number $100 \leq \mathrm{Re} \leq 1000$ will be presented.

This paper is organized as follow. In Section 2.0, the theory of the doublepopulation thermal lattice Boltzmann numerical method is presented. In the subsequent section, a detailed description of the problem is discussed. The simulated results in terms of streamlines, isotherms and local Nusselt number are shown and discussed.

\section{MATHEMATICAL FORMULATION}

For the computations, a two-dimensional (2D) double population function thermal lattice Boltzmann model originally proposed by $\mathrm{He}$ et al. (1998) with single time Bhatnagar-Gross-Krook (BGK) relaxation collision operator (He et al., 1998) is used. The governing equations for this model are defined as Eq. (1) and Eq. (2):

$$
\begin{aligned}
& f_{i}\left(x+c_{i} \Delta x, t+\Delta t\right)-f_{i}(x, t)=\frac{1}{\tau_{f}}\left(f_{i}(x, t)-f_{i}^{\theta q}(x, t)\right)+F_{f} \\
& g_{i}\left(x+c_{i} \Delta x, t+\Delta t\right)-g_{i}(x, t)=\frac{1}{\tau_{g}}\left(g_{i}(x, t)-g_{i}^{\theta q}(x, t)\right)
\end{aligned}
$$


where subscript $i$ indicates the velocity direction, and $\Delta x$ and $\Delta t$ are the lattice grid spacing and time step, respectively. The particle speed, $\boldsymbol{c}$, is defined as $\boldsymbol{c}=\frac{\Delta x}{\Delta t} \cdot f_{i}(x, t)$ and $g_{i}(x, t)$ are density and internal energy distribution function associated with the particle at node $\mathrm{x}$ and time $\mathrm{t}$ with velocity $\boldsymbol{c}_{i} \cdot f_{i}^{\in q}(x, t)$ and $g_{i}^{e q}(x, t)$ are the corresponding local equilibrium distribution functions. In this study, a 2D nine-velocity model is assigned for density distribution function, $i=1,2, \ldots, 9$, whereas the 2D fourvelocity proposed by Azwadi and Tanahashi (2008), $i=1,2,3,4$ or a nine-velocity model is used for the internal energy density distribution function, depending on the simulation conditions. The lattice structures for these models are shown in Figure 1.

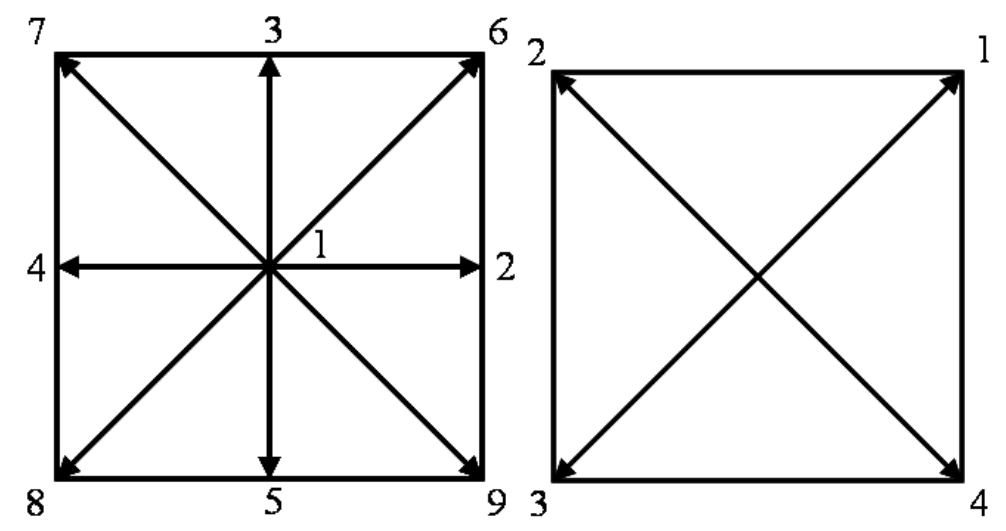

Figure 1. Lattice structure for 2D nine-velocity (left) and 2D four-velocity (right)

For the nine-velocity model, the lattice velocities are defined to be

$$
c_{i}=\left\{\begin{array}{cc}
0, & i=1 \\
( \pm 1,0),(0, \pm 1), & i=2,3,4,5 \\
( \pm 1, \pm 1), & i=6,7,8,9
\end{array}\right.
$$

The equilibrium distribution function for the density and internal energy density distribution function are given by

$$
\begin{aligned}
& f_{i}^{\boxminus q}=\rho \omega_{i}\left[1+3 c_{i} \cdot \boldsymbol{u}+\frac{9\left(c_{i} \cdot \boldsymbol{u}\right)^{2}}{2}-\frac{3 \boldsymbol{u}^{2}}{2}\right] \\
& g_{i}^{e q}=\rho \omega_{i} T\left[1+3 c_{i} \cdot \boldsymbol{u}+\frac{9\left(c_{i} \cdot \boldsymbol{u}\right)^{2}}{2}-\frac{3 u^{2}}{2}\right]
\end{aligned}
$$

where $\omega_{1}=4 / 9, \omega_{2-5}=1 / 9$ and $\omega_{6-9}=1 / 36$. For the four-velocity model, the lattice velocities are defined by

$$
c_{i}=\{( \pm 1, \pm 1), \quad i=1,2,3,4
$$

and the equilibrium distribution function for the internal energy distribution function is given by

$$
g_{i}^{\oplus q}=\rho \omega_{i} T\left[1+3 c_{i} \cdot u\right]
$$


where $\omega_{1-4}=1 / 4$. The macroscopic variables, such as density $\rho$, velocity $u$, and temperature $T$ can be evaluated as the moment to the distribution function

$$
\rho=\int f d \boldsymbol{c}_{,} \rho \boldsymbol{u}=\int \boldsymbol{c} f d \boldsymbol{c}_{y} T=\int g d \boldsymbol{c}
$$

A Chapman-Enskog expansion procedure can be applied to Eq. 1 and Eq. 2 to derive the macroscopic transport equations of the model (Azwadi, 2007). They are given by the continuity equation

the momentum equation

$$
\nabla \cdot u=0
$$

$$
\frac{\partial u}{\partial t}+u \nabla \cdot u=-\frac{1}{\rho} \nabla p+v \nabla^{2} u
$$

and the energy equation

$$
\frac{\partial T}{\partial t}+\nabla \cdot(u T)=\chi \nabla^{2} T
$$

The pressure is given by $p=c_{s}^{2} \rho$, where $c_{s}$ is the speed of sound with $c_{s}^{2}=1 / 3$. The relaxation time of internal energy carried by the particles to its equilibrium is different from that of momentum (He et al., 1998). Therefore, we differentiated the two relaxation times to characterize the momentum and energy as in Eqs. 1 and 2 by $\tau_{f}$ and $\tau_{g}$. $\tau_{f}$ can be related to the kinematic viscosity by

$$
v=\frac{2 \tau_{f}-1}{6}
$$

For the four-velocity model, $\tau_{g}$ can be related to the thermal diffusivity by

$$
\chi=\tau_{g}
$$

\section{NUMERICAL PROCEDURE}

The system of interest is a lid driven cavity with a heat source in the form of a square cylinder positioned at the vertical centre line with $0.5 \mathrm{H}$. Figure 2 shows the geometry in which mixed convective heat transfer is studied in the present work. The size of heated square, $D$ and the enclosure height, $H$ define the aspect ratio of the geometry test case (aspect ratio, $B=D / H)$. In present study, the aspect ratio was fixed at 0.2 . The boundary conditions in this investigation are as follow. The cavity walls were maintained at a constant cold temperature $T c$, whereas the inner square was maintained at a constant hot temperature $T_{H}$. The top lid cavity is moving with velocity $U_{\text {lid }}$. The physical domain between the outer and inner square is filled with air $(P r=0.71)$ as working fluid. The temperature difference between the outer and inner squares introduces a temperature gradient in a fluid, and the velocity of the top lid consequent density difference induces a fluid motion, that is, convection. The gravitational acceleration acts in the negative $y$ direction. The computational domain and specified boundary conditions are shown in Figure 3. 


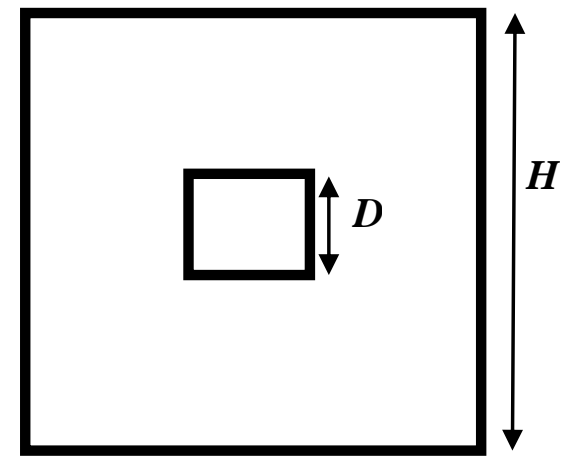

Figure 2. Schematic geometry for heated square inside cavity

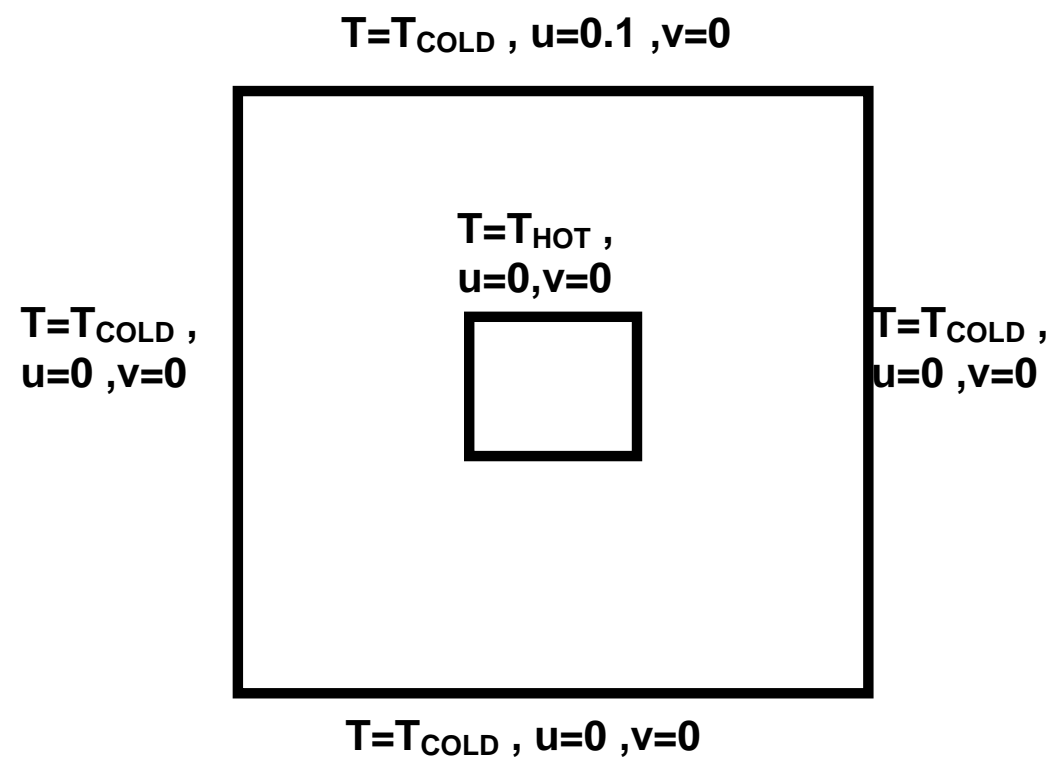

Figure 3. Computational model with boundary condition

A grid dependence test was carried out to see the effect of the size of the numerical grid on the maximum value of the vertical velocity, $\mathrm{V}_{\max }$ and the maximum horizontal velocity $\mathrm{U}_{\max }$. The simulation at $R e=100$ and $\mathrm{Ri}=10$ was selected to conduct the grid dependence test. Seven levels of grids, namely, 60, 80, 100, 120, 150, 180 and 200, were tested. As can be seen from Figure 4, as the grid is refined, the variation in the two successive grids decreases. A grid size of 150 was chosen for all subsequent Reynolds numbers with corresponding Richardson numbers by considering the relative time of computation with desirable accuracy.

The convergence criterion for all the tested cases is

$$
\begin{aligned}
& \max \left|f^{n+1}-f^{n}\right| \leq 10^{-5} \\
& \max \left|g^{n+1}-g^{n}\right| \leq 10^{-5}
\end{aligned}
$$

where the calculation is carried out over the entire system. 


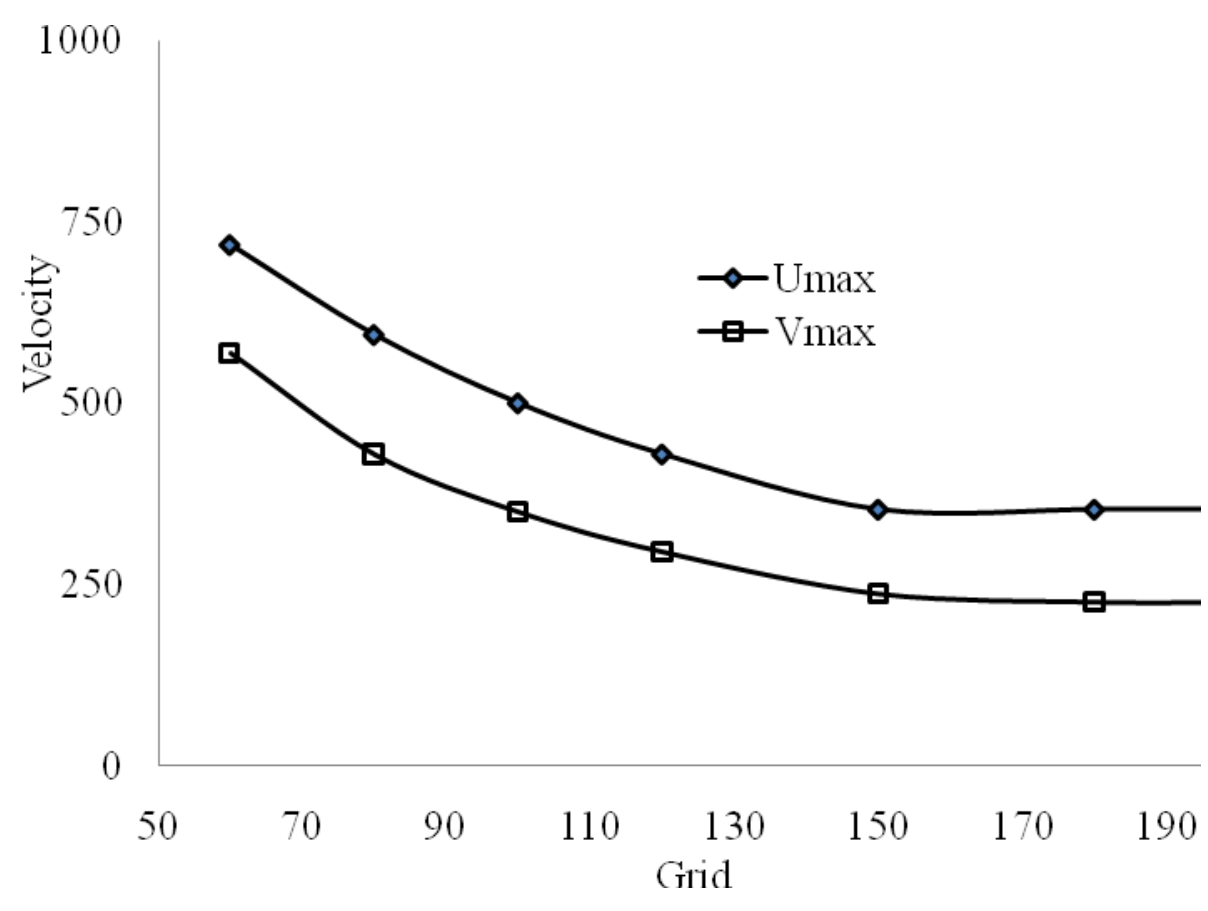

Figure 4. Grid dependency test at $\mathrm{Ri}=10$ and $\mathrm{Re}=100$.

\section{RESULTS AND DISCUSSION}

Three non-dimensional parameters playedan important role for the mixed (forcenatural) convection problem: (1) the Reynolds number $R e=\frac{U_{l i d} L}{v}$ where $\mathrm{U}_{\text {lid }}$ is the impressed top lid velocity; (2) the Richardson number $R i=G r / R e^{2}$ where $G r$ is the Grashof number $G r=\beta g \Delta T L^{3} / v^{2}$ in which $\beta$ is the coefficient of thermal expansion of the fluid, $g$ the acceleration due to gravity, and $\Delta T=T_{\text {Hot }}-T_{\text {cold }}$ the temperature difference between the inner hot square cylinder and cold cavity walls; (3) the Prandtl number $\operatorname{Pr}=v / \chi$, where $v$ is the kinematic viscosity and $\chi$ the thermal diffusivity of the fluid.

In this section, numerical results for the problem obtained from thermal lattice Boltzmann scheme are discussed for $\operatorname{Pr}=0.71$. The Reynolds number $100<\operatorname{Re}<1000$ and the corresponding Richardson number $0.01<\mathrm{Ri}<10$ were investigated numerically. We carefully chose the characteristic speed $v_{c}=\sqrt{g L \Delta T}$ so that the low Mach number approximation holds.

The predicted streamlines and isotherms in the cavity at steady state for every case are shown in Figures 5-8. In Figures 5 and 6, the Reynolds number was fixed at $\mathrm{Re}=100$ and corresponding Richardson numbers are $\mathrm{Ri}=0.01, \mathrm{Ri}=0.1, \mathrm{Ri}=1$ and $\mathrm{Ri}=10$. At $\mathrm{Ri}=0.01$, the heat transfer mechanism is dominated by force convection. The isotherm is in smooth lines and recirculation flow appeared at the top of the square cavity. The isotherms were distorted and the recirculation flow is moving to the direction of moving lid by increasing $\mathrm{Ri}=0.1$. Distortion is clearly seen at $\mathrm{Ri}=1$, where mixed convection takes place. At this stage, the isotherm is heading to the direction of moving lid and a thin thermal boundary layer is present along the moving lid. 
Secondary recirculation flow exists at the bottom right corner of the cavity and the main recirculation flow moves to the top right corner of the heated square.

For the natural convection dominated case with $\mathrm{Ri}=10$, the isotherm is distorted and the main recirculation flow is diminished. The presence of a very thin thermal boundary layer along the moving lid and others walls is noticed. Three numbers of secondary recirculation flow evolved at the three corner of the cavity.

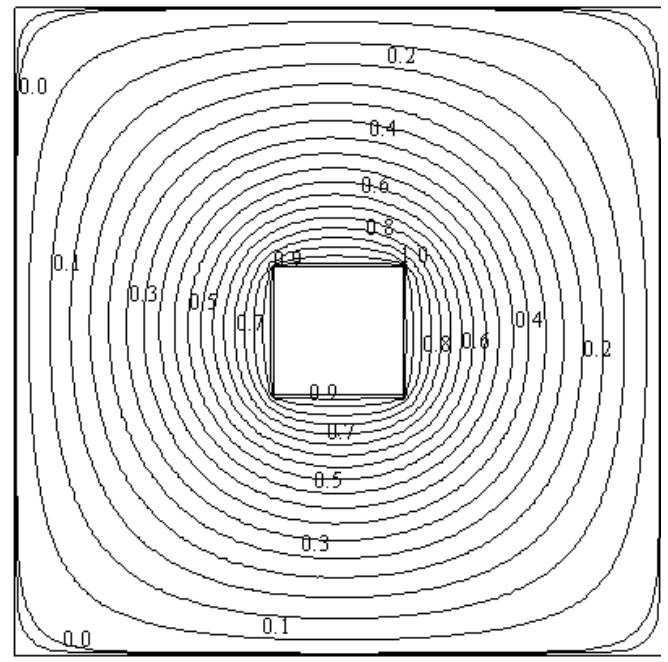

$$
\mathbf{R i}=\mathbf{0 . 0 1}
$$

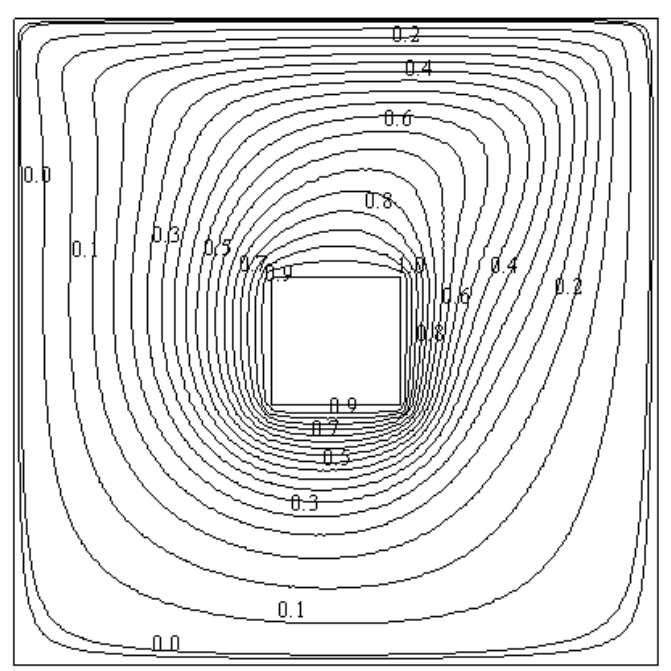

$\mathbf{R i}=\mathbf{1}$

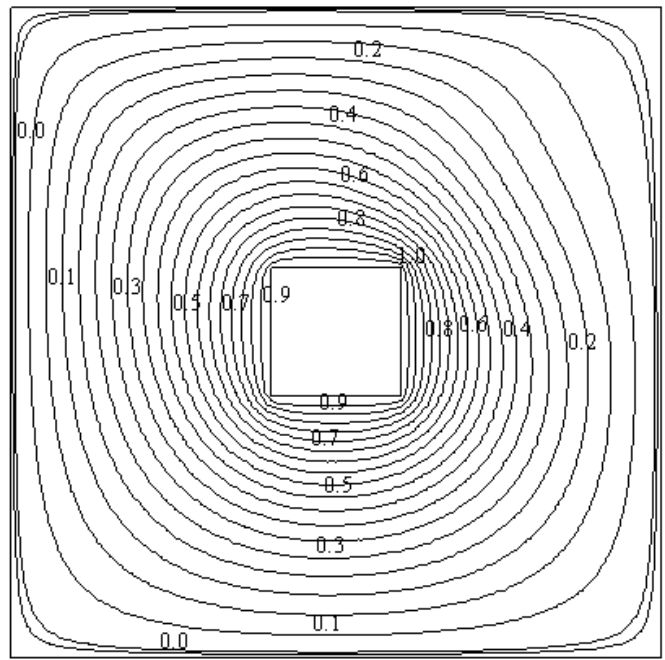

$\mathbf{R i}=\mathbf{0 . 1}$

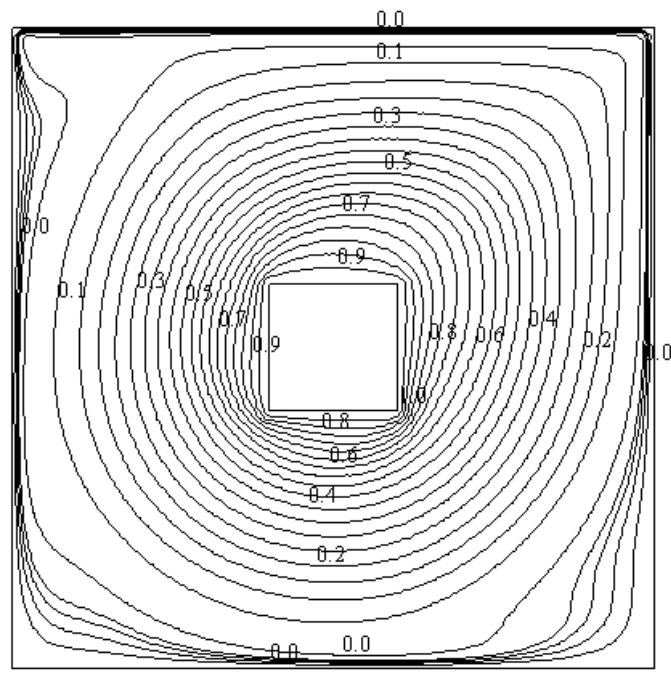

$$
\mathbf{R i}=\mathbf{1 0}
$$

Figure 5. Isotherm for different Richardson numbers at $\mathrm{Re}=100$. 


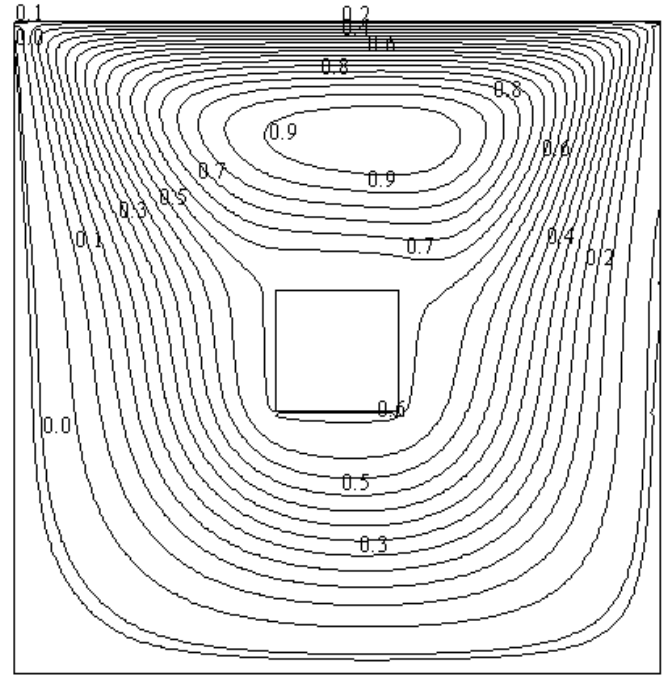

$\mathbf{R i}=\mathbf{0 . 0 1}$

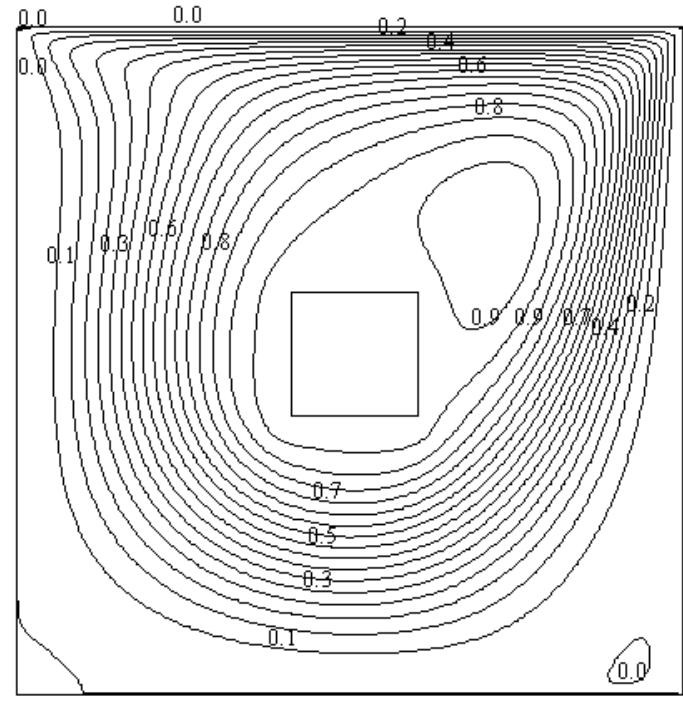

$\mathbf{R i}=\mathbf{1}$

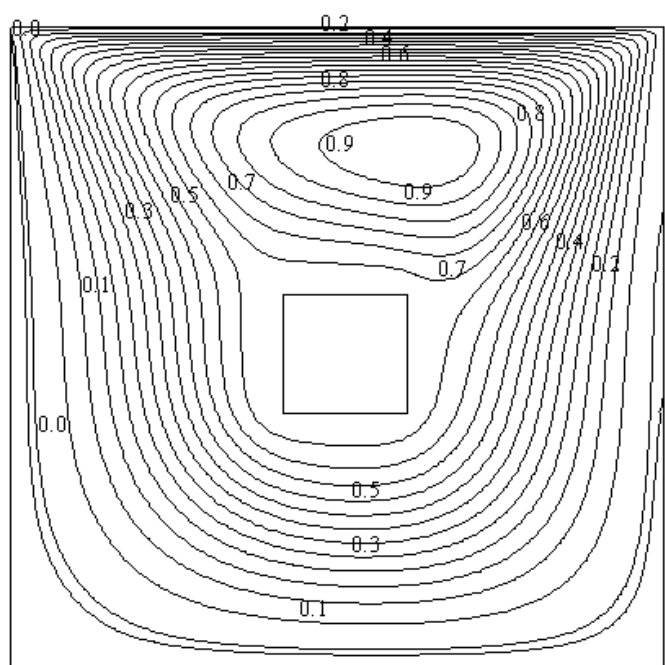

$\mathbf{R i}=\mathbf{0 . 1}$

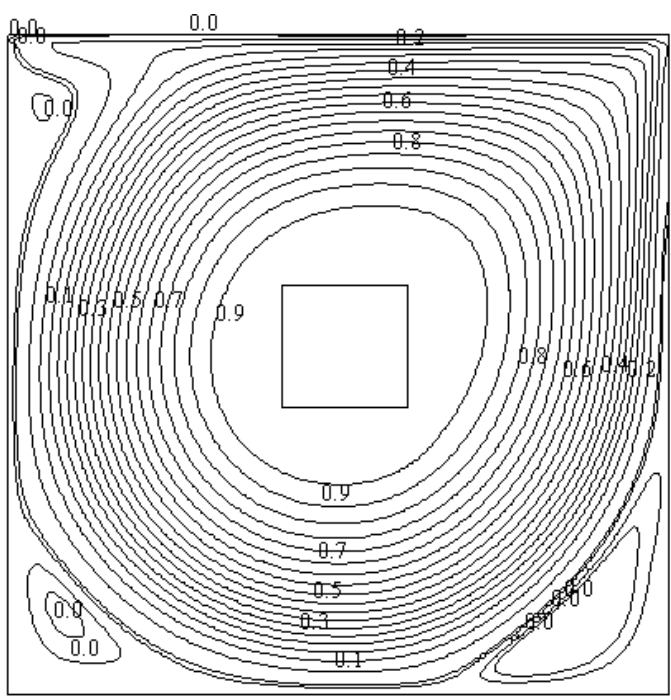

$\mathbf{R i}=\mathbf{1 0}$

Figure 6. Streamlines for different Richardson numbers at $\mathrm{Re}=100$.

Figures 7 and 8 shows the results for various Reynolds numbers $100<\operatorname{Re}<1000$ at the fixed Richardson number $\mathrm{Ri}=0.01$. The isotherm was distorted by increasing the Reynolds number and distortion heads in the direction of the moving lid. By increasing the Reynolds number, a secondary recirculation flow is produced at the bottom right corner of the cavity and the main recirculation flow moves to the top right corner of the heated square. 
Simulation of mixed convective heat transfer using lattice Boltzmann method

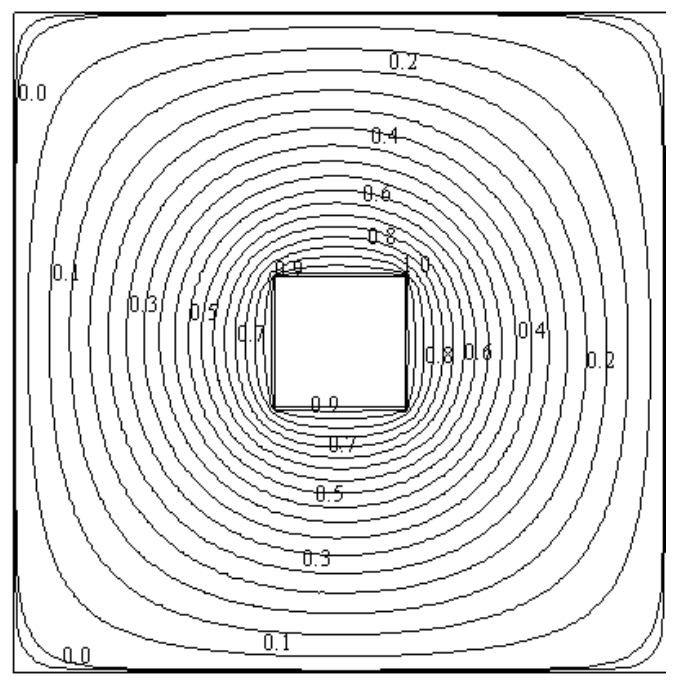

$\operatorname{Re}=100$

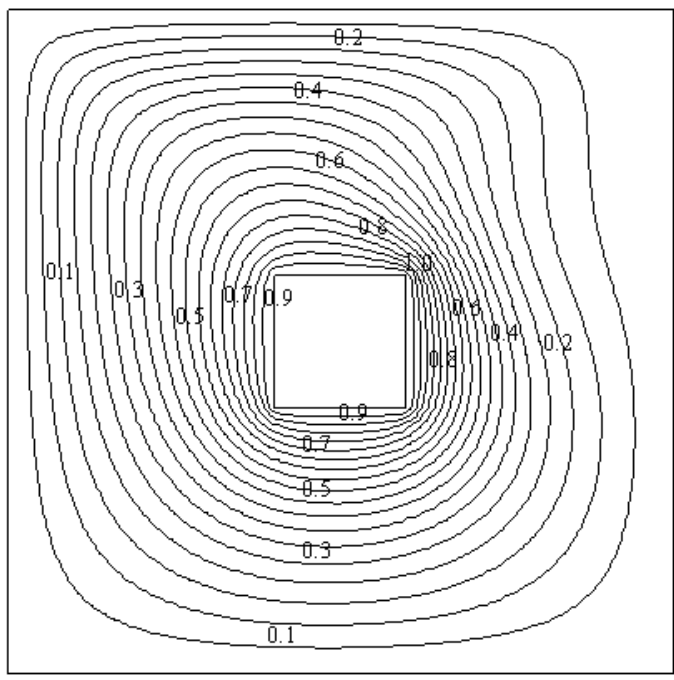

$\operatorname{Re}=400$

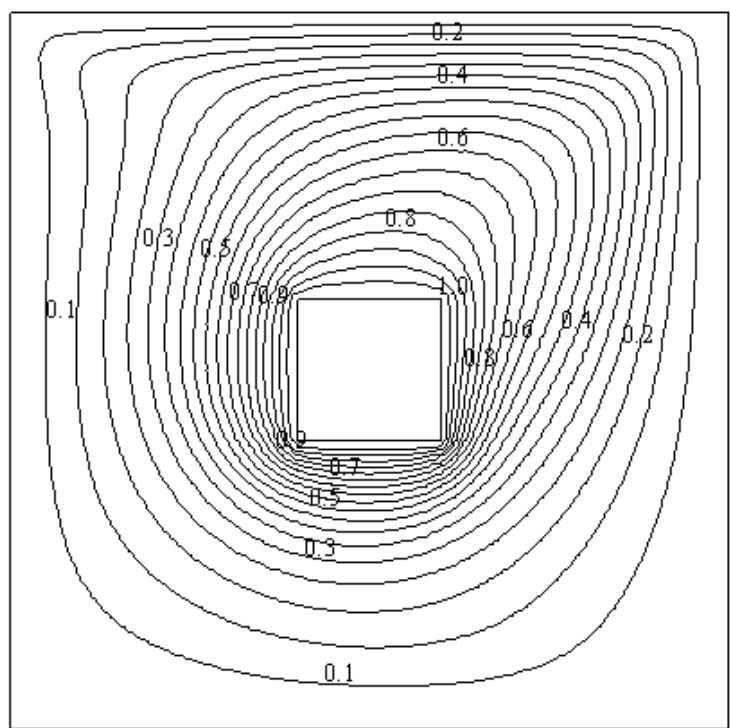

\section{$\operatorname{Re}=\mathbf{1 0 0 0}$}

Figure 7. Isotherms for different Reynolds numbers at $\mathrm{Ri}=0.01$. 


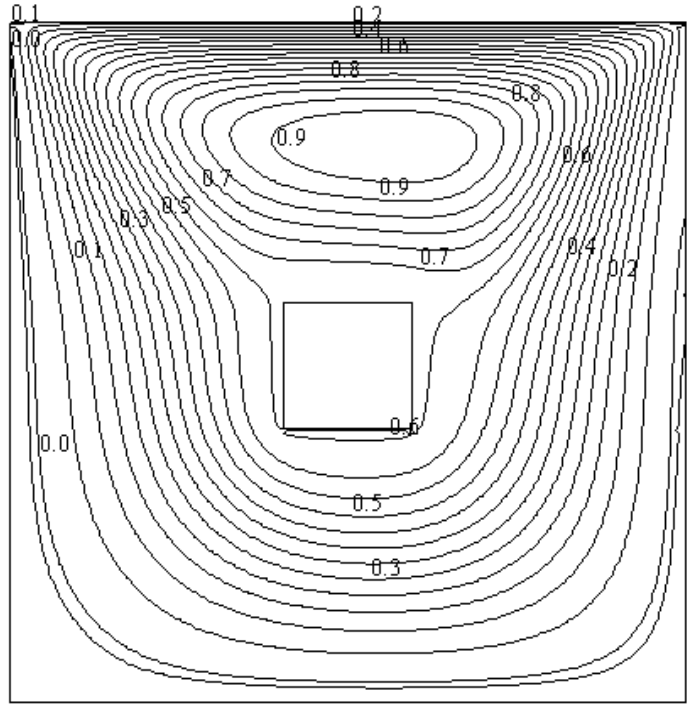

$\operatorname{Re}=100$

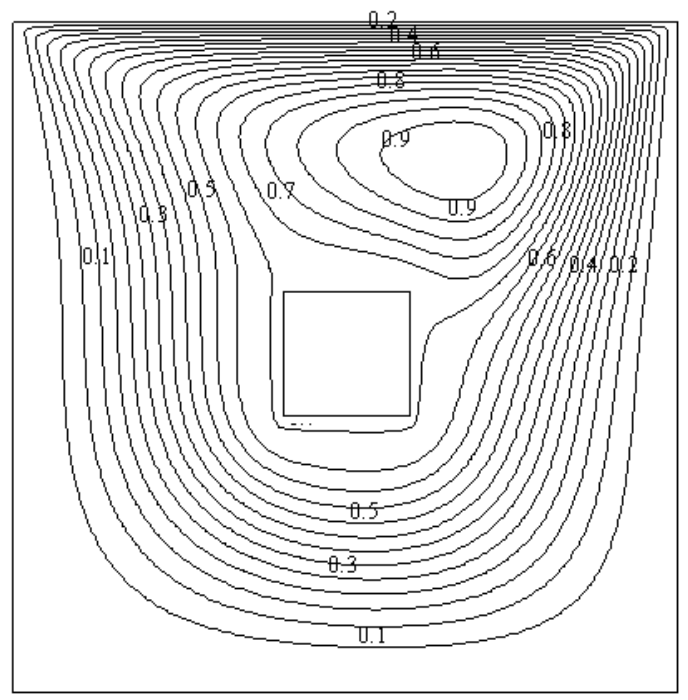

$\operatorname{Re}=400$

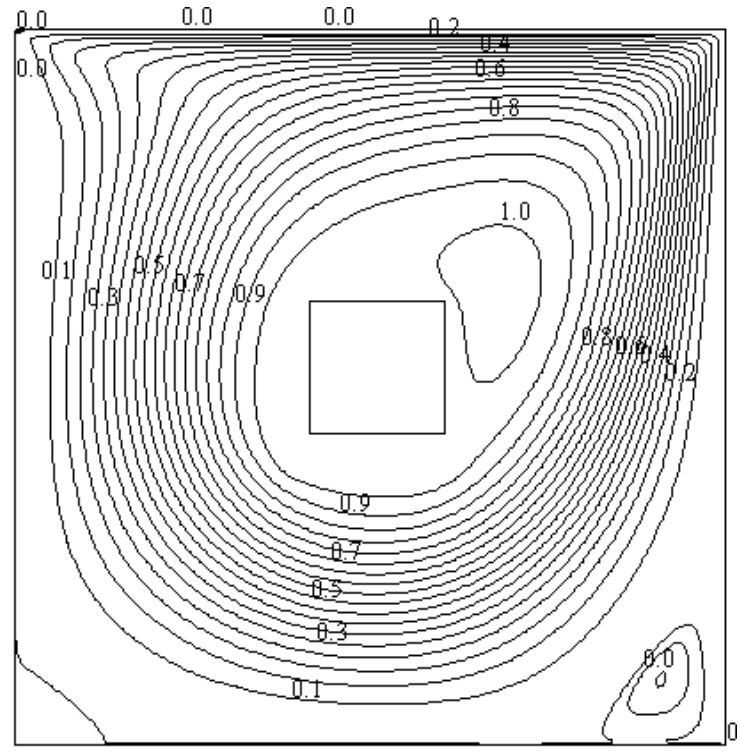

$\operatorname{Re}=1000$

Figure 8. Streamlines for different Reynolds numbers at Ri=0.01.

The variation of the local Nusselt number along the top surface of the heated square is plotted in Figures 9 and 10 for all cases studied. The effect of the Richardson number and Reynolds number is clearly discernible in these plots. In general, the Nusselt number starts with a high value at the left end and decreases to a small value and increases towards the right end. At any location the Nusselt number decreases with increasing Richardson number at $\mathrm{Re}=100$ and also decreases with increasing Reynolds number at $\mathrm{Ri}=0.01$. 


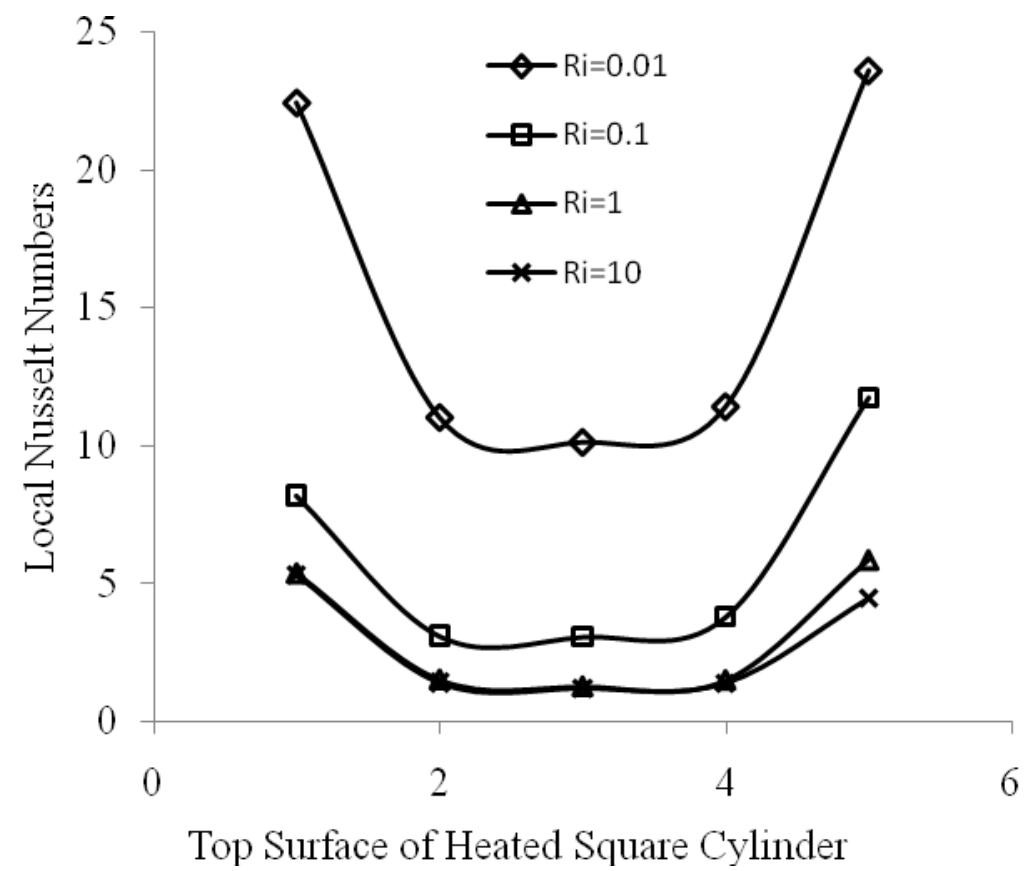

Figure 9: Local Nusselt number on top surface of heated square at different Richardson numbers.

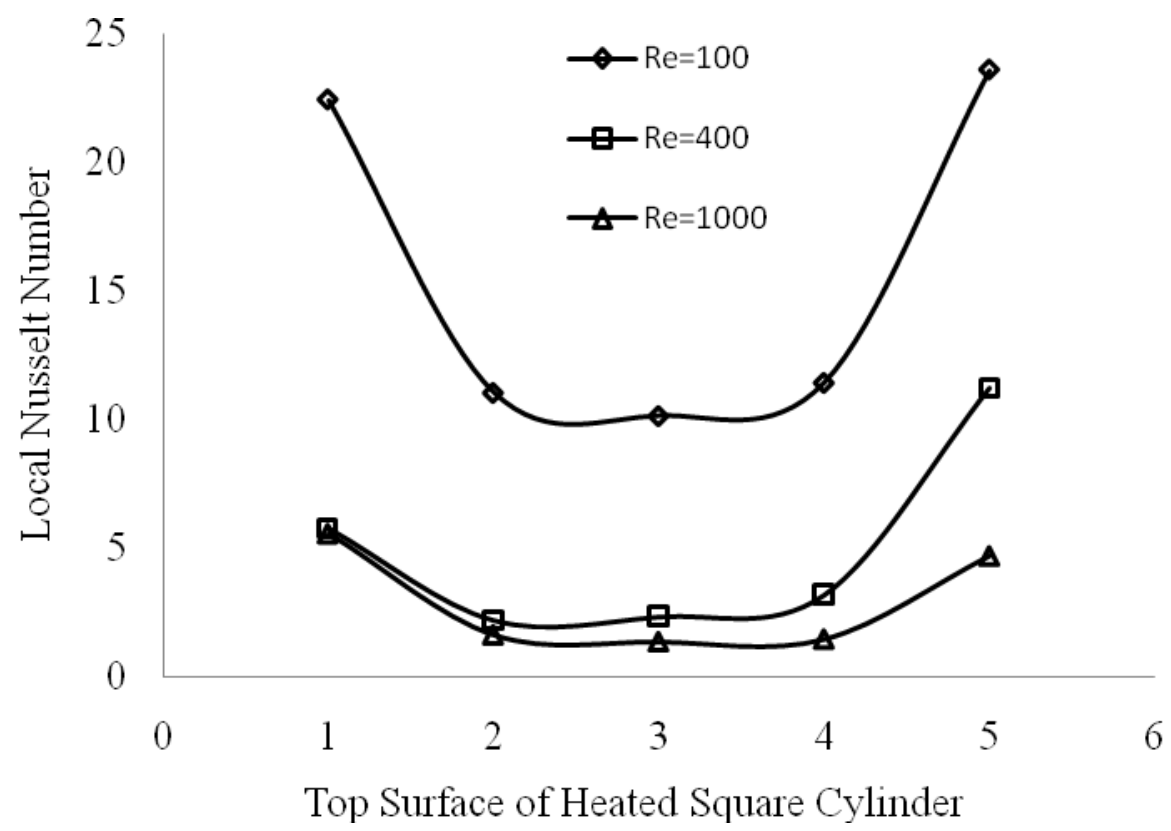

Figure 10: Local Nusselt number on top surface of heated square at different Reynolds numbers.

In Figures 11 and 12, as the Richardson number increases to $\mathrm{Ri}=10$, the dimensionless horizontal and vertical velocity profile given at the mid height and mid width of the cavity gradually increases the velocity slightly from the centre to the four side walls and narrow boundary layers develop along the walls. The peak values of both horizontal velocity and vertical velocity are due to the intensified convective activities 
from increasing the Richardson number. Increasing the Richardson number is proportional to the increase of the buoyancy force effect. Both figures show the different velocity directions for every wall, these directions correspond to the development of recirculation flow.

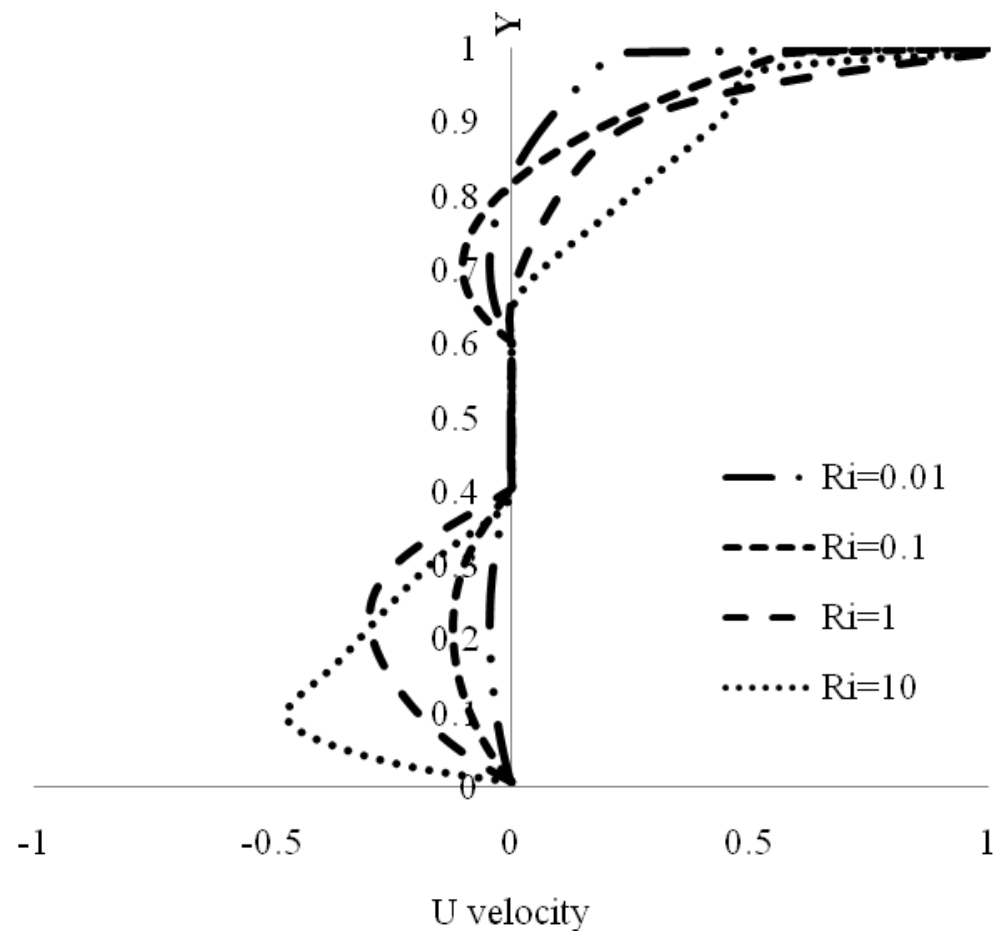

Figure 11: Velocity profiles along the vertical mid-plane in the cavity.

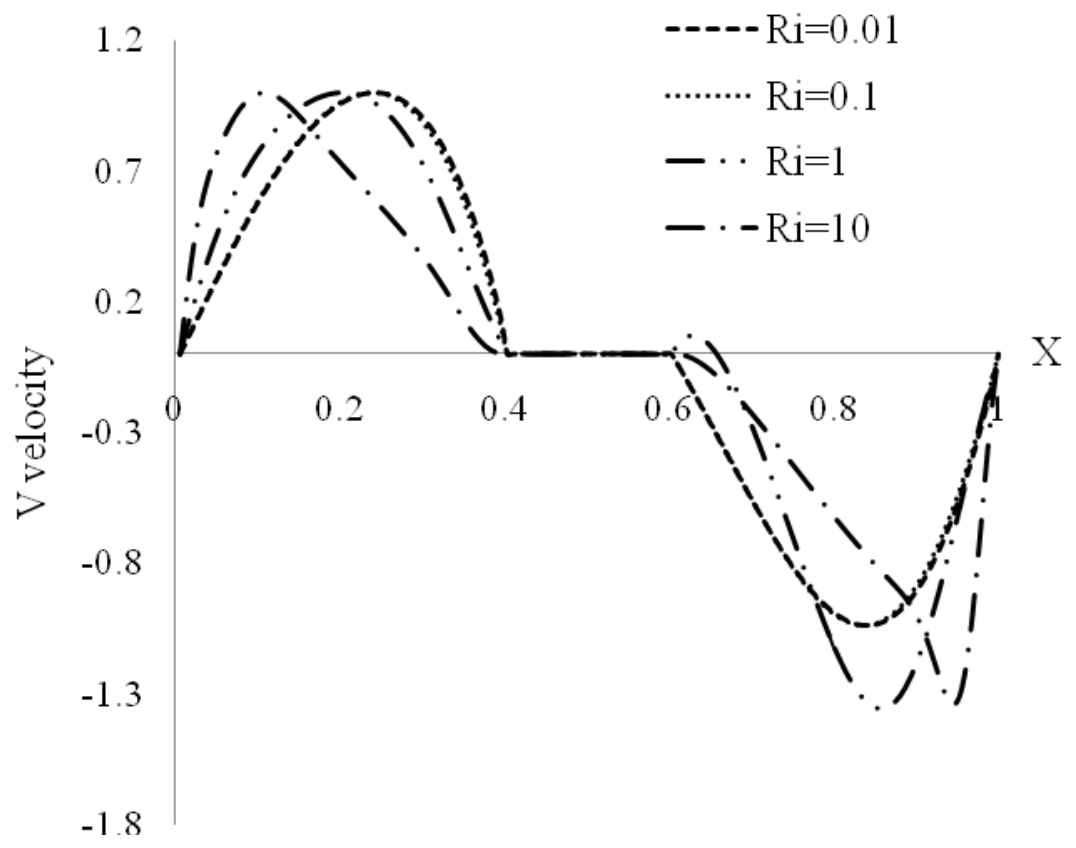

Figure 12: Velocity profiles along the horizontal mid-plane in the cavity. 


\section{CONCLUSION}

A mathematical model was developed to analyse the two-dimensional lid driven cavity with a heated square of mixed (forced-natural) convective heat transfer using the lattice Boltzmann method. The result shows the capability of the lattice Boltzmann method to analyse mixed convective heat transfer problems. The main parameters of interest are the Reynolds number, Richardson number and Prandtl number. The variation of the Nusselt number along the top surface of the heated square was presented. The advantage of the lattice Boltzmann method (microscopic level) over the directly solved NavierStokes equation (macroscopic level) is the Knudsen number. This research can be extended to simulate heat transfer problem with low Knudsen number.

\section{ACKNOWLEDGEMENTS}

The authors wish to thank the Universiti Pertahanan Nasional Malaysia and the Malaysia Government for supporting this research activity.

\section{REFERENCES}

Abdalla, M.A., Khanafer, K., Bull, J., and Pop, I. (2007) Effect of Sinusoidal Wavy Bottom Surface on Mixed Convection Heat Transfer in a Lid-Driven Cavity. International Journal of Heat and Mass Transfer 50: 1771-1780.

Abdelkhalek, M.M. (2008) Mixed Convection in a Square Cavity by a Perturbation Technique. Computational Materials Science 42: 212-219.

Angeli, D., Paolo, L., and Giovanni, S. (2008) Numerical Prediction for Stable Buoyant Regimes Within a Square Cavity Containing a Heated Horizontal Cylinder. International Journal of Heat and Mass Transfer, 51: 553-565.

Arnab, K., and Amaresh, D. (2006) A Numerical Study of Natural Convection Around a Square, Horizontal, Heated Cylinder Placed in an Enclosure. International Journal of Heat and Mass Transfer, 49: 4608-4623.

Azwadi, C.S.N. (2007)The Development of Simplified Thermal Lattice Boltzmann Models for the Simulation of Thermal Fluid Flow Problems. PhD Thesis, Keio University, Japan.

Azwadi, C.S.N., and Tanahashi, T. (2008) Simplified Finite Difference Thermal Lattice Boltzmann Method.International Journal of Modern Physics B, 22: 3865-3876.

He, X., Shan, S., and Doolen, G. D. (1998) A Novel Thermal Model for the Lattice Boltzmann Method in Incompressible Limit.Journal of Computational Physics 146: 282-300.

Khanafer, K.M., Abdalla, M.A., and Pop, I. (2007) Numerical Simulation of Unsteady Mixed Convection in a Driven Cavity Using an Externally Excited Sliding Lid. European Journal of Mechanics B/Fluids, 26: 669-687.

Kim, B.S., Lee, D.S., Haa, M.Y., and Yoon, H.S. (2008) A Numerical Study of Natural Convection in a Square Enclosure With a Circular Cylinder at Different Vertical Locations. International Journal of Heat and Mass Transfer, 51: 1888-1906.

Ouertatani, N., Cheikh, N.B., Beya, B.B., Lili, T., and Campo, A. (2009) Mixed Convection in a Double Lid-Driven Cubic Cavity. International Journal of Thermal Sciences, 48: 1265-1272. 
Oztop, H.F., Zhao, Z., and Yu, B. (2009) Fluid Flow Due to Combined Convection in Lid-Driven Enclosure Having a Circular Body. International Journal of Heat and Fluid Flow 30: 886-901.

Oztop, H.F., and Dagtekin, I. (2004) Mixed Convection in Two-Sided Lid-Driven Differentially Heated Square Cavity. International Journal of Heat and Mass Transfer 47: 1761-1769.

Sharif, M.A.R. (2007) Laminar Mixed Convection in Shallow Inclined Driven Cavities With Hot Moving Lid on Top and Cooled From Bottom. Applied Thermal Engineering, 27: 1036-1042.

Shohel, M., Prodip, K.D., and Nasim, H. (2002) Laminar Natural Convection Around an Isothermal Square Cylinder at Different Orientations. International Communication on Heat and Mass Transfer, 29: 993-1003.

Waheed, M.A. (2009) Mixed Convective Heat Transfer in Rectangular Enclosures Driven by a Continuously Moving Horizontal Plate. International Journal of Heat and Mass Transfer, 52: 5055-5063.

\section{Nomenclature}

$\boldsymbol{B} \quad$ aspect ratio $(\boldsymbol{B}=\mathrm{D} / \mathrm{H})$

$\boldsymbol{c}$ particle speed

$D \quad$ height of the heated square cylinder, $m$

$f_{i} \quad$ density distribution function

$F_{f} \quad$ external force

$g \quad$ accelerationdue to gravity, $\mathrm{ms}^{-2}$

$g_{i} \quad$ Internal energy distribution function

Gr Grashof number based on $H$ and $T_{H}-T_{C}$

$H$ height of the cavity, m

$k$ thermal conductivity of air, $\mathrm{W} \mathrm{m}^{-1} \mathrm{~K}^{-1}$

$L \quad$ length of the cavity, $m$

$P \quad$ dimensionless local pressure

Pr Prandtl number

$\mathrm{Ra} \quad$ Rayleigh number

Re Reynolds number based on $H$ and $U_{\text {lid }}$

$\mathrm{Ri} \quad$ Richardson number

$T_{C} \quad$ temperature of the cold surface, $\mathrm{K}$

$T_{H} \quad$ temperature of the hot surface (lid), $\mathrm{K}$

$U$ dimensionless velocity component in $x$-direction

$U_{\text {lid }} \quad$ lid velocity, $\mathrm{ms}^{-1}$

$V \quad$ dimensionless velocity component in $y$-direction

$\alpha \quad$ thermal diffusivity, $\mathrm{m}^{2} \mathrm{~s}^{-1}$

$\beta \quad$ thermal expansion coefficient, $\mathrm{K}^{-1}$

$\theta$ dimensionless temperature

$\rho$ density, $\mathrm{kg} \mathrm{m}^{-3}$

$\tau_{f}, \tau_{g}$ time relaxation

$v \quad$ kinematic viscosity, $\mathrm{m}^{2} \mathrm{~s}^{-1}$

$\omega_{i} \quad$ weighting function

$\chi \quad$ thermal diffusivity, $\mathrm{m}^{2} \mathrm{~s}^{-1}$ 